\title{
A Performance Comparative Analysis of Block Based Compressive Sensing and Line Based Compressive Sensing
}

\author{
Mansoor Ebrahim \\ Department of Computer Science \\ Iqra University \\ Karachi, Pakistan \\ mebrahim@iqra.edu.pk
}

\author{
Syed Hasan Adil \\ Department of Computer Science \\ Iqra University \\ Karachi, Pakistan \\ hasan.adil@iqra.edu.pk
}

\author{
Daniyal Nawaz \\ Department of Computer Science \\ Iqra University \\ Karachi, Pakistan \\ daniyal.nawaz@iqra.edu.pk
}

\begin{abstract}
Compressive sensing (CS) is an innovative idea that has opened new areas for viable communication of correlated data. In this paper, a comparative performance analysis of two different variants of compressive sensing i.e. block based compressive sensing (BCS) and line based compressive censing (LCS) schemes is performed for natural images. The idea is to evaluate which variant performs better in terms of reconstruction quality and provides easy initial solution. The experimental analysis demonstrates that LCS scheme can enhance the image reconstruction at lower subrates by $0.5 \mathrm{~dB}$ to $2.5 \mathrm{~dB}$, when compared to the BCS scheme.
\end{abstract}

Keywords-compressive sensing; block based approach; line based approach; reconstruction; image

\section{INTRODUCTION}

Compressive sensing (CS) is one of the latest techniques that have achieved popularity recently and is applied to various imaging applications [1], such as magnetic resonance imaging (MRI) [2, 3] and seismic identification [4]. CS [5, 6] idea is to express a signal (image/video) with sample estimation rate much lower than that of the Nyquist rate which is essential for the recovery of the signal. One of the innovative of this is the single-pixel camera [1] that openly condenses the sampling and amount of data that will be transmitted, but increases the difficulty of recovering the original signal. In other words, the recovery of the original signal from small number of sample measurements is difficult. Numerous earlier researches have already examined the utilization of CS in image compression [3-8]. Many researchers concentrated their work on proposing how natural images can be compressed utilizing CS and the recovery of the encoded signal (image) from such little estimation. In CS we concentrate mostly towards discrete signs instead of constant time space signals. In addition, compressive sensing of natural images is exposed to few issues such as computationally complex reconstruction algorithm and requirement of large memory to store the random sampling operator $\Phi$. In order to encounter the aforementioned issue different approaches were developed i.e. block based compressive sensing and line based compressive sensing. The block-based approach is far more developed and widely employed as compared to the newly developed line based approach to reduce of the computationally complexity of CS scheme [9-14]. Such methodologies value CS because (i) block/line based estimation is more convenient for applications where the sample image data don't need to be encoded completely in a block/line form until the point when the estimation of the whole image is completed, (ii) the application and capacity of the estimation operator are straightforward, (iii) the individual handling of each block/line of image data brings about simple initial solution with considerably quick and better recovery process.

In this paper, a performance comparison of two different variants of compressive sensing i.e. block based compressive sensing and line based compressive sensing for images is performed. The purpose is to find out which variant works best to solve the issues related to CS. The variants are evaluated based on computational complexity and reconstruction quality at various subrates for different test image datasets. In addition, block based and line based approaches are also compared at various block/line sizes to validate the effectiveness of each scheme. The paper is structured as follows. The fundamentals of the CS along with block based and line based approaches are presented in section II. Section III presents and discusses the simulation results. Section IV concludes the paper.

\section{THEORY OF COMPRESSIVE SENSING}

The CS theory [5] states that a sparse (original or some transform domain) signal can be completely recovered from a number of samples below than that stated by the Nyquist theorem. The CS scheme efficiently eases the computational prerequisites, for example, memory, processing power, and transmission data transfer capacity at the encoder by relating signal acquirement and dimensionality reduction into a distinct stage. CS is effective in two circumstances. When direct measurements of a high-resolution signal are hard to attain and when multiple high-resolution signals are complex to encode. In literature, CS is a standard and isn't stated for any particular signal other than underlying sparsity assumptions. CS permits great prospect of signal reconstruction by utilizing least amount of unsystematic measurements, as long as the signal/image is 
sparse and incoherent. Unlike conventional compression schemes, in CS the encoder only captures the signal measurements rather than the whole signal. This helps in reducing the computational complexity and bandwidth.

Consider a real-valued signal $\mathrm{X}$ with length $\mathrm{N}$ from $\mathrm{M}$ measurementsis to be recovered, has certain sparsity in the transformation domain $\Psi$ with random measurement matrix $\Phi$ as shown in Figure 1. The set of measurements y is given as:

$$
\mathrm{Y}=\Phi \cdot \mathrm{X}
$$

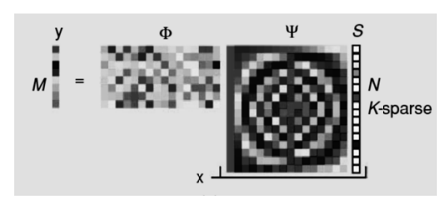

Fig. 1. Compressive sensing acquisition process with a random measurement matrix $\Phi$ and transformation matrix $\Psi$ [6]

$X \in R_{N}$ and $Y \in R_{M}$ are the input signal and measurement vector respectively. It is presumed that the random sensing matrix $\Phi$ is orthonormal i.e. $\Phi \Phi^{\mathrm{T}}=\mathrm{A}$, where $\mathrm{A}$ is the identity matrix. In addition, the recovery of $\mathrm{X}$ from such small estimation is not straight forward, i.e. inverse projection of $\mathrm{X}=\Phi^{-1} \mathrm{Y}$ is ill-posed [6]. But since the signal to be compressed by CS ought to be sparse in nature, the recovery can be completed by taking care of the $\ell_{0}$ optimization problem that can be expressed as:

$$
\hat{\mathrm{x}}=\operatorname{argmin}_{\hat{\mathrm{x}}}\|\hat{\mathrm{x}}\|_{\text {lo }} \text {, s.t. } \mathrm{y}=\Phi \Psi-1 \mathrm{X},
$$

However, most of existing works [3-8] in CS remain at the theoretical and are exposed to few issues, like that at encoder CS requires accessing the whole signal for sampling simultaneously that makes its real time sensing of natural signals difficult. At decoder, the reconstruction of such sampled signals is generally very expensive. To counter this issue, various researchers proposed different variants of CS that mainly include block based CS, and line based CS.

\section{A. Block Based Compressive Sensing}

Block based CS [9] technique has been proposed to exploit the sparse nature of images in transform domain in order to reduce storage space. In block based CS the original image is divided in terms of blocks and each block is processed separately based on the similar measurement matrix " $\Phi$ " with a constrained (block-diagonal) structure as in (3).

$$
\Phi=\left[\begin{array}{ccc}
\Phi_{\mathrm{B}} & \cdots & 0 \\
\vdots & \ddots & \vdots \\
0 & \cdots & \Phi_{\mathrm{B}}
\end{array}\right]
$$

The key pluses of using BCS scheme are that (i) the sampled image data do not need to be transmitted by the encoder until the measurement of the whole image is done i.e. making block-based measurement more expedient for practical applications, (iii) each image block is processed individually to bring information about simple preliminary arrangement with primarily quick reconstruction process [10]. The BCS based reconstruction implicates two different variants i.e. smooth projection land-weber (SPL) and total variation (TV) minimization. This paper focuses on TV minimization scheme as it provides better reconstruction compared to SPL and finds the evenest course within the potential space by making usage of piece-wise smooth properties of typical signals instead of finding the sparse solution within the transformation domain $\Psi$. The reconstruction process of BCS is discussed in section II.C.

\section{1) Encoder}

Suppose, an image (I) has $\mathrm{I}_{\mathrm{R}}$ (Rows) $\mathrm{x} \mathrm{I}_{\mathrm{C}}$ (Columns) pixels, $\left(I=I_{R} \cdot I_{C}\right)$. The image $I$ is divided into different small blocks, each block having size of $B \cdot B$. Let $X_{B}$ represent the vectorized signal of the $b$-th block through raster scanning. The compressed output for this b-th block will be:

$$
\mathrm{Y}_{\mathrm{B}}=\Phi \cdot \mathrm{X}_{\mathrm{B}}
$$

For the complete image, each block is then independently sampled by the similar measurement matrix $\Phi$ with a constrained arrangement. The encoded sampled $\mathrm{Y}$ are then communicated to the decoder for recovery. Summarizing, the encoding steps are:

- Input image $\mathrm{I}$ of size $\mathrm{I}_{\mathrm{R}} \cdot \mathrm{I}_{\mathrm{C}}$.

- Convert image I into multiple blocks X.

- $\quad$ Sampled each block $X_{i}$ with Gaussian Matrix $\Phi$.

- $\quad$ Finally, Y will be the encoded sample.

The encoding process of block based compressive sensing is presented in Figure 2.

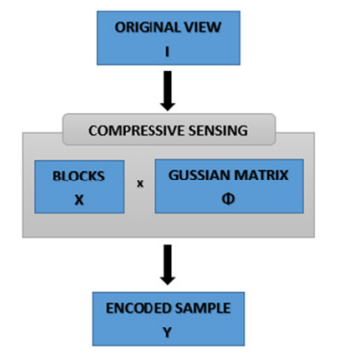

Fig. 2. Encoding process of BCS

\section{B. Line Based Compressive Sensing}

Line based CS $[15,16]$ is a recent approach in which the original image is first divided into $\mathrm{N}$ multiple lines of same size rather than in blocks. Each line is then processed independently using the measurement operator $\Phi$. This approach benefits CS because in line based approach sampled signal does not need to be encoded as a whole but in a line by line fashion independently. In addition, each independent line of the signal results in easy initial solution with considerably fast and improved recovery process. The LCS based reconstruction is based on TV minimization scheme as it provides fast and better reconstruction of the encoded measurements. The reconstruction process of LCS is discussed in section II.C.

\section{1) Encoder}

Consider an $\mathrm{I}_{\mathrm{R}} \cdot \mathrm{I}_{\mathrm{C}}$ image, where $\mathrm{I}_{\mathrm{R}}$ and $\mathrm{I}_{\mathrm{C}}$ represent the pixel values in each row and column respectively. At encoder side, 
the image is first distributed into $\mathrm{N}$ multiple lines, using the line based CS. Each line will have a size of $1 \cdot \mathrm{L}$ and is processed independently using the measurement operator $\Phi$. Consider $\mathrm{X}_{\mathrm{i}}$ as the vectorized signal of $\mathrm{i}$-th line of the image. Then the compressed CS vector output $\mathrm{Y}_{\mathrm{i}}$ will be:

$$
\mathrm{Y}_{\mathrm{L}}=\Phi \cdot \mathrm{X}_{\mathrm{L}}
$$

For the entire image, each line is then separately sampled utilizing a similar estimation matrix $\Phi$ with a constrained structure. The estimations $\mathrm{Y}$ are then transmitted to the decoder for recovery. The encoding process of line based compressive sensing is presented in Figure 3 and its steps are.

- Input image $\mathrm{I}$ of size $\mathrm{I}_{\mathrm{R}} \times \mathrm{I}_{\mathrm{C}}$.

- Convert image I into multiple lines X.

- Sampled each line $X_{i}$ with Gaussian Matrix $\Phi$.

- Finally, Y will be the encoded sample

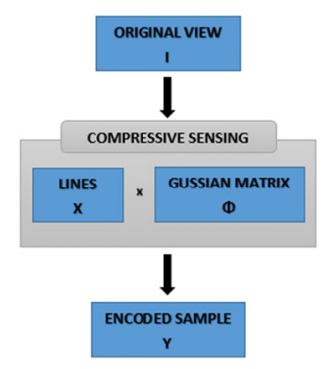

Fig. 3. Encoding process of LCS

\section{Decoding}

The reconstruction of the encoded estimations is the primary challenge of utilizing CS. As the number of unknown elements is higher than the number of observations, recovery of $X \in R_{N}$ from its relating $Y \in R_{M}$ i.e. inverse projection of $X=\Phi^{-1} \cdot Y$ is ill-posed [6]. Since the signal to be compressed by CS ought to be sparse in nature, the recovery can be completed by taking care of convex optimization problem using sparsity in transformed domain with either $\ell$-norm or image gradient with total variation (TV) norm [7]. The TV minimization finds the smoothest arrangement inside the potential space by making utilization of piece-wise smooth qualities of natural signals as opposed to finding the inadequate arrangement inside the transformation domain. The essential TV minimization function is given as:

$$
\begin{aligned}
& \mathrm{TV}(\mathrm{X})=\sum \mathrm{i}, \mathrm{j}\|\mathrm{Xi}+1, \mathrm{j}-\mathrm{Xi}, \mathrm{j}\|+\|\mathrm{Xi}, \mathrm{j}+1-\mathrm{Xi}, \mathrm{j}\| \\
& \min \mathrm{X} \mathrm{E}\|\mathrm{y}-\Theta \mathrm{X}\|+\lambda \mathrm{TV}(\mathrm{X}) \text { s.t. } \Theta=\Phi \Psi
\end{aligned}
$$

However, the TV minimization based CS reconstruction problem in (7) is exposed to extra computational burdens, i.e. the non-differentiable (presence of the absolute value function) and non-linear properties, restricting its use for CS reconstruction. In [17], a scheme named as TV-AL3 is proposed to solve (7). The scheme is a combination of conventional augmented Lagrangian method with variable splitting and the alternating direction method. TV-AL3 generates reconstructed image with the high quality of standard $\mathrm{TV}$, but reduces the computational burden by applying splitting and alternating approaches. The purpose of splitting is to distinct the non-differentiable terms from the differentiable terms, so as to facilitate low-complexity sub-problems in an alternating minimization way [18], resulting in decreased computational burden. Moreover, the augmented Lagrangian method of TV-AL3 varies from the typical Lagrangian method by adding a square penalty term, whereas from the quadratic penalty method by the presence of the linear term involving the multiplier $\lambda$ [18]. The generic decoding process for the reconstruction of encoded measurements produced by BCS and LCS is presented in Figure 4 and its steps are:

- Input the encoded sample Y and parameters $(\Phi, \mathrm{R}, \mathrm{C}$, block size/line size, etc.)

- Reconstruct encoded sample Y with the help of plenty parameter by solving (5) using total variation augmented Lagrangian (TV-AL3).

- Finally, I' will be the final reconstructed view of the actual image I.

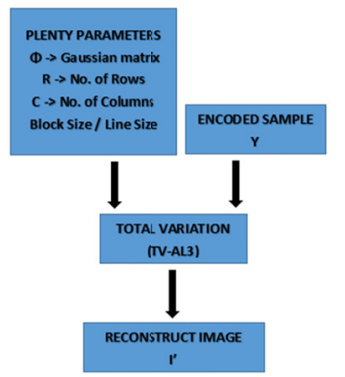

Fig. 4. Decoding process of BCS and LCS

\section{EXPERIMENTAL RESULTS}

In this section the performance evaluation of block based and line based CS are presented. A set of test images is used to evaluate the schemes performance. For the work reported in this paper six images, shown in Figure 5, are used, obtained from [19-21]. The selected dataset consist of images with various characteristics such as high and low percentage of untextured surface, variations and disparity ranges to evaluate their performances in different conditions. To make the images amenable by both schemes, we down sampled the original images size to $512 \times 512$ pixels. The down sampled images are challenging in contrast to standard due to their larger disparity range and higher percentage of un-textured surface. The performance comparison involves using the two different variants of compressive sensing. The variants (BCS, LCS) were implemented by using the algorithms proposed in $[9,16]$ respectively. We also investigated the effect of block size on the image reconstruction quality. The evaluation is carried on two different block/line sizes $(32 \times 32 / 1 \times 1024$ and $64 \times 64 / 1 \times 4096)$ by measuring the peak-signal to noise-ratio (PSNR) at various subrates. The idea is to analyze the impact of block/line size and subrate on the reconstruction quality of the encoded measurements obtained by both the discussed schemes (BCS, LCS). Additionally, as the measurement matrix 
$\Phi$ is random in nature, the image reconstruction quality might vary. Hence, an average of 5 independent trials of all PSNR values is presented. All images are sampled at lower subrates (0.05-0.3) with an interval of 0.05 .

\section{A. $B C S$ vs $L C S$}

In this section, the performance of BCS is compared with LCS. The comparison is to investigate the rate-distortion (R-D) performance of BCS and LCS. All R-D performances' (i.e., PSNR (dB), subrate) results are obtained from the six mentioned images by using different block/line sizes at various subrates. Table I presents the comparison results of LCS and BCS for the images and the two different sizes considered. From the simulation results, it is observed that the LCS scheme shows an average gain $1 \mathrm{~dB}-3 \mathrm{~dB}$ for lower to higher subrates over BCS scheme and the gain is more prominent in low variation image, Middle-burry. Furthermore, from [22] it is stated that larger measurement size provides better reconstruction quality at the expense of complexity. Figures 6 and 7 show the average PSNR results of the six images for LCS and BCS at two different block/line sizes as discussed above.
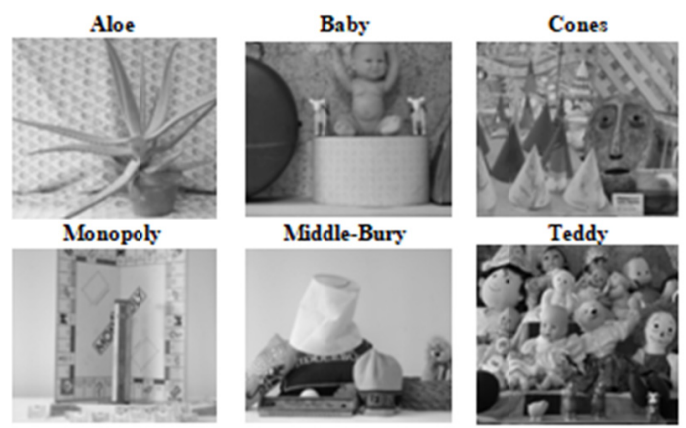

Fig. 5. Several standard grayscale test image datasets of size $512 \times 512$

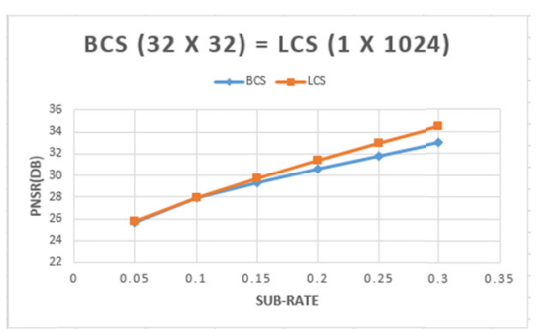

Fig. 6. Average PSNR results of six different images with block/line size of $32 \times 32 / 1 \times 1024$

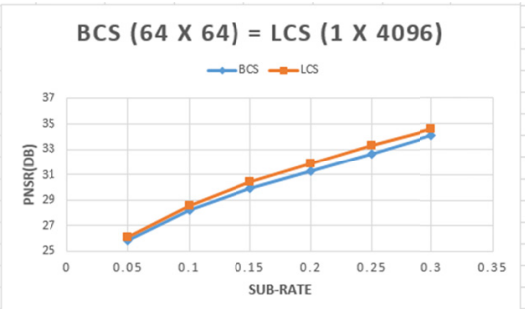

Fig. 7. Average PSNR results of six different images with block/line size of $64 \times 64 / 1 \times 4096$
TABLE I. PSNR ACCOMPLISHED BY UTILIZING BCS AND LCS TO ENCODE VARIOUS DATASET IMAGES AT BLOCK/LINE SIZE OF 32X32/1X1024

\begin{tabular}{|c|c|c|c|c|c|c|}
\hline \multicolumn{7}{|c|}{ Aloe } \\
\hline Subrate & 0.05 & 0.1 & 0.15 & 0.2 & 0.25 & 0.3 \\
\hline BCS & 24.35 & 25.25 & 26.18 & 27.19 & 28.15 & 32.69 \\
\hline LCS & 24.85 & 25.89 & 26.87 & 28.01 & 29.12 & 33.87 \\
\hline Gain & 0.5 & 0.64 & 0.69 & 0.82 & 0.97 & 1.18 \\
\hline \multicolumn{7}{|c|}{ Baby } \\
\hline Subrate & 0.05 & 0.1 & 0.15 & 0.2 & 0.25 & 0.3 \\
\hline BCS & 28.98 & 30.68 & 31.99 & 33.01 & 34.73 & 35.89 \\
\hline LCS & 29.75 & 31.48 & 33 & 34.08 & 35.84 & 37.03 \\
\hline Gain & 0.77 & 0.8 & 1.01 & 1.07 & 1.11 & 1.14 \\
\hline \multicolumn{7}{|c|}{ Monopoly } \\
\hline Subrate & 0.05 & 0.1 & 0.15 & 0.2 & 0.25 & 0.3 \\
\hline BCS & 25.91 & 28.03 & 29.66 & 31.74 & 34.17 & 35.1 \\
\hline LCS & 26.74 & 28.94 & 30.69 & 32.84 & 35.57 & 36.57 \\
\hline Gain & 0.83 & 0.91 & 1.03 & 1.1 & 1.4 & 1.47 \\
\hline \multicolumn{7}{|c|}{ Middle-burry } \\
\hline Subrate & 0.05 & 0.1 & 0.15 & 0.2 & 0.25 & 0.3 \\
\hline BCS & 25.79 & 28.99 & 31.14 & 32.98 & 34.54 & 35.66 \\
\hline LCS & 26.65 & 29.89 & 32.15 & 34.21 & 35.8 & 36.98 \\
\hline Gain & 0.86 & 0.9 & 1.01 & 1.23 & 1.26 & 1.32 \\
\hline \multicolumn{7}{|c|}{ Cones } \\
\hline Subrate & 0.05 & 0.1 & 0.15 & 0.2 & 0.25 & 0.3 \\
\hline BCS & 24.7 & 26.98 & 28.57 & 29.84 & 31.08 & 32.07 \\
\hline LCS & 25.83 & 28.27 & 30.12 & 31.76 & 33.31 & 34.65 \\
\hline Gain & 1.13 & 1.29 & 1.55 & 1.92 & 2.23 & 2.58 \\
\hline \multicolumn{7}{|c|}{ Teddy } \\
\hline Subrate & 0.05 & 0.1 & 0.15 & 0.2 & 0.25 & 0.3 \\
\hline BCS & 24.96 & 26.99 & 29.05 & 29.77 & 30.89 & 32.07 \\
\hline Gain & 26.02 & 28.18 & 30.29 & 31.34 & 33.16 & 34.51 \\
\hline
\end{tabular}

\section{B. Visual Result Comparison}

Since LCS and BCS are evaluated at lower subrates, it is essential to certify that the subrates used are adequate to create visually detectable images. Two different image datasets are selected representing medium and high texture variations. The results shown in Figures 8 and 9 are of the reconstructed images of the encoded measurements obtained from BCS and LCS using two different subrates, 0.1 and 0.2 . It can be noticed that the reconstructed images from LCS improve the distorting effect present in the image reconstructed using the BCS encoded measurements. Also, by comparing the emphasized regions (red dotted boxes), it can be noticed that reconstruction performed by using LCS encoded measurements looks much smoother and piercing than the reconstruction of BCS measurements.
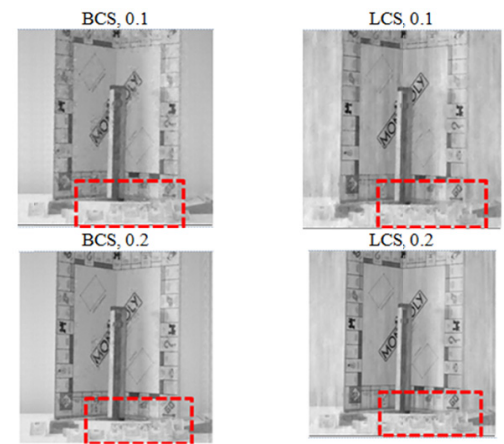

Fig. 8. Visual results of the reconstruction of Monopoly image using BCS and LCS encoded measurements at different subrates 

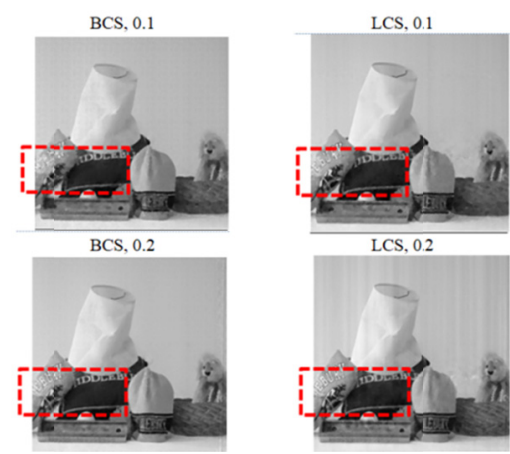

Fig. 9. Visual results of the reconstruction of Middle-burry image using BCS and LCS encoded measurements at different subrates.

\section{CONCLUSION}

This article presents a performance comparison between block based compressive sensing and line based compressive sensing for different image datasets encoded at various subrates and sizes. The experimental result verifies that the line based approach performs better than the block based approach by $1 \mathrm{~dB}$ to $3 \mathrm{~dB}$ at various sub-rates and sizes for different images.

\section{REFERENCES}

[1] M. F. Duarte, M. A. Davenport, D. Takhar, J. N. Laska, T. Sun, K. F. Kelly, R. G. Baraniuk, "Single pixel imaging via compressive sampling”, IEEE Signal Processing Magazine, Vol. 25, No. 2, pp. 83-91, 2008

[2] M. Lustig, D. L. Donoho, J. M. Santos, J. M. Pauly, "Compressed Sensing MRI”, IEEE Signal Processing Magazine, Vol. 25, No. 2, pp. 72-82, 2008

[3] M. Lustig, D. L. Donoho, J. M. Pauly, "Sparse MRI: The Application of compressed sensing in Rapid MRI imaging", Magnetic Resonance in Medicine, Vol. 58, No. 6, pp. 1182-1195, 2007

[4] W. Tang, J. Ma, F. J. Herrmann, Optimized CS for Curve-Let Based Seismic Data Reconstruction, preprint, 2008

[5] D. L. Donoho, "Compressed sensing", IEEE Transactions on Information Theory, Vol. 52, No. 4, pp. 1289-1306, 2006

[6] E. J. Candes, M. B. Wakin, "An introduction to compressive sampling", IEEE Signal Processing Magazine, Vol. 25, No. 2, pp. 21-30, 2008

[7] A. Chambolle, P. L. Lions, "Image recovery via total variation minimization and related problems", Numerische Mathematik, Vol. 76, No. 2, pp. 167-188, 1997

[8] M. A. T. Figueiredo, R. D. Nowak, S. J. Wright, “Gradient projection for sparse reconstruction: application to compressed sensing and other inverse problems", IEEE Journal of Selected Topics in Signal Processing, Vol. 1, No. 4, pp. 586-597, 2007

[9] L. Gan, "Block compressed sensing of natural images", 15th International Conference on Digital Signal Processing, Cardiff, UK, pp. 403-406, July 1-4, 2007

[10] S. Mun, J. E. Fowler, "Block Compressed Sensing of Images Using Directional Transforms", 16th IEEE International Conference on Image Processing, Cairo, Egypt, pp. 3021-3024, November 7-10, 2009

[11] J. E. Fowler, S. Mun, E. W. Tramel, "Block-based compressed sensing of images \& video", Foundations \& Trends in Signal Processing, Vol. 4, No. 4, pp. 297-416, 2012

[12] F. Shi, J. Cheng, L. Wang, P.-T. Yap, D. Shen, "Low-Rank Total Variation for Image Super-Resolution", Lecture Notes in Computer Science, Vol. 8149, pp. 155-162, 2013

[13] M. Ebrahim, C. W. Chong, "Multi-view Image compression for Visual Sensor Network based on Block Compressive Sensing and multi-phase join decoding”, International Conference on Computational Science and Technology, Kota Kinabalu, Malaysia, August 27-28, 2014
[14] M. Ebrahim, C. W. Chong, "Multiview Image Block Compressive Sensing with Joint Multiphase Decoding for Visual Sensor Network", ACM Transactions on Multimedia Computing, Communications, and Applications, Vol. 12, No. 2, Article No. 30, 2015

[15] T. F. Chan, S. Esedoglu, F. Park, A. Yip, "Total variation image reconstruction: overview and recent developments", in: Handbook of Mathematical Models in Computer Vision, Springer, pp. 17-31, 2005

[16] M. Ebrahim, D. Nawaz, S. H. Adil, "Line based Compressive Sensing for low power application”, Press Electronic World, 2017

[17] C. Li, An efficient algorithm for total variation regularization with applications to the single pixel camera and compressive sensing, MSc Thesis, Rice University, USA, 2010

[18] C. Li, Compressive sensing for $3 \mathrm{~d}$ data processing tasks: applications, models and algorithms, PhD Thesis, Rice University, USA, 2013

[19] C. Strecha, R. Fransens, L. Van Gool, "Combined depth and outlier estimation in multi-view stereo", IEEE Computer Society Conference on Computer Vision and Pattern Recognition, New York, USA, pp. 23942401, June 17-22, 2006

[20] D. Scharstein, R. Szeliski, "High-accuracy stereo depth maps using structured light", IEEE Computer Society Conference on Computer Vision and Pattern Recognition, Madison, USA, Vol. 1, pp. 195-202, June 18-20, 2003

[21] L. M. Po, CityU Image Database, Retrieved January 12, 2014, from http://abacus.ee.cityu.edu.hk/imagedb/dbroot/Stereo_Image

[22] Y. Baig, E. M. K. Lai, A. Punchihewa, "Distributed video coding based on compressed sensing", IEEE International Conference on Multimedia and Expo Workshops (ICMEW), Melbourne, Australia, pp. 325-330, July 9-13, 2012 